\title{
Percepciones sobre la educación de personas adultas. Análisis de la formación, la práctica docente y el perfil profesional
}

\author{
Marisol Rodríguez Correa \\ Universidad Internacional de la Rioja. España. \\ marisol.rodriguez@unir.net \\ Juan-Carlos Rivadulla-López \\ Universidade da Coruña. España. \\ juan.rivadulla@udc.gal
}

Recibido: $13 / 4 / 2016$

Aceptado: 24/5/2016

Publicado: 26/10/2017

\section{Resumen}

En los últimos años, la educación y la formación de adultos ha adquirido tal desarrollo que cada vez son más las personas cuya actividad profesional gira en torno a este ámbito. Sin embargo, en una sociedad incierta y altamente competitiva en la que se demandan más actividades formativas, el profesorado de dicha disciplina sigue sin recibir la formación específica necesaria para enfrentarse a esa realidad. Este vacío formativo ha sido una de las principales razones que nos han impulsado a querer conocer, desde la voz de sus protagonistas, las percepciones que tienen los profesores de educación de personas adultas (EPA) sobre su labor pedagógica, para así tratar de identificar un perfil docente de los mismos. En esta investigación, participaron 90 enseñantes de EPA de Galicia (España), y la recogida de datos se realizó mediante un cuestionario tipo Likert. Los resultados muestran que los docentes consideran que la EPA es poco valorada por la sociedad, que requieren mayor formación específica y que no creen que su labor sea una profesión.

Palabras clave: educación de adultos; formación continua; personal docente; carrera profesional

Resum. Percepcions sobre l'educació de persones adultes. Anàlisi de la formació, la pràctica docent $i$ el perfil professional

En els últims anys, l'educació i la formació d'adults ha adquirit un desenvolupament tan gran que cada vegada són més les persones l'activitat professional de les quals gira entorn d'aquest àmbit. No obstant això, en una societat incerta i altament competitiva en què es demanen més activitats formatives, el professorat d'aquesta disciplina segueix sense rebre la formació específica necessària per enfrontar-se a aquesta realitat. Aquest buit formatiu ha estat una de les raons principals que ens han fet voler conèixer, directament des dels seus protagonistes, les percepcions que tenen els professors d'educació de persones adultes (EPA) sobre la seva labor educativa, per així tractar d'identificar-ne un perfil docent. En aquesta investigació, hi van participar 90 ensenyants d'EPA de Galícia (Espanya), i la 
recollida de dades es va realitzar mitjançant un qüestionari tipus Likert. Els resultats mostren que els docents consideren que l'EPA és poc valorada per la societat, que necessiten més formació específica i que no troben que la seva tasca sigui una professió.

Paraules clau: educació d'adults; formació contínua; personal docent; carrera professional

Abstract. Perceptions of adult education. Analysis on training, educational practice and professional profile

In recent years, adult education and training has become widespread, with an increasing number of people working in the field. However, in an uncertain and highly competitive society that demands more training activities, adult education teachers still do not receive the specific training they require. Due to the lack of training in this field, the aim of this study was to determine the perceptions of adult education teachers regarding their work in order to identify their teaching profiles. Ninety adult education teachers of Galicia, Spain, participated in the study from whom data were collected using a Likert questionnaire. The results show that teachers believe that adult education is undervalued by society. Moreover, they request more specific training and do not consider their work as a profession.

Keywords: adult education; continuing training; teaching personnel; career

\begin{aligned} & \multicolumn{2}{c}{ Sumario } \\ & 1. Introducción 4. Metodología \\ & 2. Formación de los educadores de 5. Resultados \\ & personas adultas 6. Conclusiones y discusión \\ & $\begin{aligned} \text { 3. La profesionalización del educador de } & \text { Referencias bibliográficas } \\ \text { personas adultas } & \end{aligned}\end{aligned}$

\section{Introducción}

La necesidad de vincular la formación a todo el ciclo vital de los sujetos es uno de los enfoques más interesantes que se ha adoptado en los últimos años. A partir de este planteamiento, cuando hablamos de formación, se trasciende la etapa escolar y los contenidos convencionales académicos, con lo que se constituye un proceso íntimamente unido a la realización personal y profesional de las personas (Zabalza, 2002).

A partir de la idea de que los sujetos se forman a lo largo de su vida, se ha ido configurando un nuevo marco de condiciones de tipo estructural, curricular y organizativa, entre otras, para desarrollar la formación.

Nos encontramos indudablemente ante una nueva toma de conciencia de las condiciones marcadas por el desarrollo social y económico vigente. Quizás por ese origen más vinculado a las actuales exigencias de la producción industrial y al impacto generado por las nuevas tecnologías, los más sensibles a esta necesidad de formación continuada sean los sistemas productivos, por una parte, y el mundo de la ciencia y su desarrollo imparable, por otra. Aprender 
a lo largo de la vida significa aprovechar todas las oportunidades que ofrece la sociedad, una sociedad educativa en la que toda circunstancia puede ser ocasión para adquirir conocimientos nuevos y mejorar las competencias personales, de modo que el aprendizaje es una parte intrínseca de la propia vida: en cualquier lugar y en cualquier momento las personas nos podemos instruir (Arbués y Tarín, 2000: 54). Tal y como señala Savelli (2014), nuestra sociedad impulsa al adulto a aprender por sí mismo, lo que conllevará un autocontrol, un rediseño y un trabajo constante de reformación.

Según González (2010), la educación de personas adultas tiene la finalidad de ofrecer a todos los mayores de dieciocho años la posibilidad de adquirir, actualizar, completar o ampliar sus conocimientos y sus aptitudes para su desarrollo personal y profesional, mediante:

- La adquisición de una formación básica y el acceso a las distintas enseñanzas del sistema educativo.

- La mejora de la cualificación profesional y la preparación para otras profesiones.

- El desarrollo de las capacidades personales y la participación ciudadana.

- La oportunidad de incrementar y actualizar sus competencias.

- La prevención y la resolución de conflictos personales, familiares y sociales, fomentando la igualdad efectiva de derechos y oportunidades entre hombres y mujeres.

— La corrección de riesgos de exclusión social, especialmente en los sectores más desfavorecidos.

Por lo tanto, la educación de adultos pretende mejorar la cualificación de personas que se han quedado desfasadas, ofreciéndoles la adquisición de competencias clave que exigen las profundas transformaciones sociales, y se considera «imprescindible para la restauración del carácter moral y ético en las relaciones sociales y su contribución en la formación de una verdadera ciudadanía democrática» (Rumbo, 2016: 105).

Por lo tanto, la educación de adultos deberá ser entendida como un enriquecimiento personal y cultural, a través del cual la persona desarrollará su autonomía y su espacio vital, y, a su vez, deberá ser

[...] reinterpretada desde una racionalidad crítica que busca la emancipación y el cuestionamiento de la realidad. Una realidad en la que todos somos parte y sujetos partícipes y, consecuentemente, tenemos la capacidad para poder cambiarla y transformarla en una realidad más justa, porque el mundo lo construimos entre todos. (Rumbo, 2013: 189)

La formación es imprescindible para poder desempeñar cualquier clase de trabajo, y en el caso de los educadores de personas adultas, aún más, porque es un campo que presenta muchas carencias formativas. Según la investigación de Rose, Jeris y Smith (2005): «se ha demostrado que muchos educadores de adultos trabajan en esta área sin la preparación académica suficiente» 
(p. 1305). De hecho, hay que reconocer que las oportunidades de formación específica para el profesorado de dicha disciplina han sido siempre muy escasas. La única modalidad formativa para docentes de adultos existente hasta épocas recientes es a consecuencia de la interacción con sus compañeros. Se podría hipotetizar que, en la actualidad, esta sigue siendo la vía formativa mayoritaria.

Bajo estos supuestos previos, hemos querido preguntar al profesorado de educación de personas adultas de nuestra comunidad autónoma cómo percibe y cómo vive dicha problemática. Para ello, se seleccionó una muestra representativa de la población de los educadores y las educadoras de personas adultas que imparten docencia en los institutos de titularidad pública de la comunidad autónoma de Galicia.

\section{Formación de los educadores de personas adultas}

Los educadores y las educadoras de personas adultas, tal y como han demostrado Rose et al. (2005), trabajan en esta área sin la preparación académica suficiente. Organizaciones tales como la UNESCO, en la mayoría de sus conferencias internacionales sobre esta temática, han reconocido la insuficiente formación del profesorado que se dedica a dicho ámbito y han plasmado entre sus recomendaciones la necesidad de preparar mejor a estos profesionales.

Es importante destacar, tal y como señala Rumbo (2010), que los docentes de personas adultas «no son consumidores de formación, sino sujetos activos que buscan alternativas y soluciones a los problemas que la realidad educativa les plantea» (p. 6).

En el contexto español, hemos tenido dos grandes marcos legislativos para regular el tema que estamos tratando: la Ley Orgánica General del sistema educativo (LOGSE) (1990) y la Ley Orgánica de educación (LOE) (2006). La LOE (2006) indicó la necesidad que tienen todas las personas de educarse a lo largo de la vida. La última y vigente Ley Orgánica para la mejora de la calidad educativa (LOMCE) (2013) se hace eco igualmente de este derecho, completando algunos artículos de la LOE (2006) y exponiendo, en su capítulo IX, entre otros, los siguientes objetivos y principios de la educación de personas adultas:

1. La educación de personas adultas tiene la finalidad de ofrecer a todos los mayores de dieciocho años la posibilidad de adquirir, actualizar, completar o ampliar sus conocimientos y aptitudes para su desarrollo personal y profesional.

2. Para el logro de la finalidad propuesta, las administraciones educativas podrán colaborar con otras administraciones públicas con competencias en la formación de adultos y, en especial, con la Administración laboral, así como con las corporaciones locales y los diversos agentes sociales.

3. La educación de personas adultas tendrá los siguientes objetivos: 
a) Adquirir una formación básica, ampliar y renovar sus conocimientos, habilidades y destrezas de modo permanente y facilitar el acceso a las distintas enseñanzas del sistema educativo.

b) Mejorar su cualificación profesional o adquirir una preparación para el ejercicio de otras profesiones.

c) Desarrollar sus capacidades personales, en los ámbitos expresivos, comunicativo, de relación interpersonal y de construcción del conocimiento.

d) Desarrollar su capacidad de participación en la vida social, cultural, política y económica y hacer efectivo su derecho a la ciudadanía democrática.

e) Desarrollar programas que corrijan los riesgos de exclusión social, especialmente de los sectores más desfavorecidos.

f) Responder adecuadamente a los desafíos que supone el envejecimiento progresivo de la población asegurando a las personas de mayor edad la oportunidad de incrementar y actualizar sus competencias.

g) Prever y resolver pacíficamente los conflictos personales, familiares y sociales. Fomentar la igualdad efectiva de derechos y oportunidades entre hombres y mujeres, así como analizar y valorar críticamente las desigualdades entre ellos.

h) Adquirir, ampliar y renovar los conocimientos, habilidades y destrezas necesarias para la creación de empresas y para el desempeño de actividades e iniciativas empresariales.

4. Las personas adultas pueden realizar sus aprendizajes tanto por medio de actividades de enseñanza, reglada o no reglada, como a través de la experiencia, laboral o en actividades sociales, por lo que se tenderá a establecer conexiones entre ambas vías y se adoptarán medidas para la validación de los aprendizajes así adquiridos. (LOMCE, 2013: 94)

Como podemos comprobar, se trata de una ley muy ambiciosa para el ámbito que nos ocupa, especialmente si tenemos en cuenta que nuestra memoria histórica nos recuerda que, hasta el momento, todas las directrices políticas sobre educación de las personas adultas acaban convirtiéndose en un catálogo de intenciones y deseos nunca del todo realizables, sobre todo en el caso de la formación y profesionalización de sus docentes, que sigue descansando en su voluntariedad para formarse.

Hay que reconocer que las oportunidades de formación específica para los educadores de personas adultas han sido siempre muy escasas, puesto que han dejado en manos de los propios educadores la voluntad de seguir formándose. En los últimos años, tanto la Administración central como las administraciones autonómicas se han esforzado en realizar planes específicos de formación para docentes de personas adultas a través de cursos universitarios de postgrado y de máster.

Tal y como señala Morón (2014), existe abundante investigación sobre la formación del profesorado en las etapas de infantil, primaria, secundaria, etc., pero muy poco sobre formación del profesorado y de adultos.

A partir de los datos arrojados en la investigación de Rose et al. (2005), podemos indicar que «poca gente ha participado en estudios sobre enseñanza 
para adultos. La mayoría de la gente no ve la enseñanza para adultos como una carrera permanente, sino como algo dentro del amplio campo de la educación en general» (p. 1306).

La única modalidad formativa para docentes de adultos existente hasta épocas recientes se produce a consecuencia de la interacción con sus compañeros, aprendiendo unos de otros. Por lo tanto, se podría hipotetizar que, en la actualidad, esta sigue siendo la mayor fuente de formación.

No obstante, no sería justo dejar de reconocer que la incorporación de la universidad a la formación de educadores de personas adultas ha sido un gran revulsivo, ya que se han generado procesos enriquecedores de colaboración entre las universidades y los grupos de educadores, lo que puede permitir que se produzca paulatinamente un cambio en el desarrollo profesional de este colectivo. Un cambio sin duda alguna muy necesario, ya que hoy día tenemos la visión de un mundo más humano, el cual es posible gracias a estas prácticas realizadas en nuestra sociedad para ayudar a los adultos a adquirir los conocimientos, las habilidades y los valores necesarios para llevar una vida productiva y satisfactoria (Watkins y Marsick, 2014).

Un dato a tener en cuenta en la educación de personas adultas es el tema del voluntariado y su formación. Así, los planteamientos formativos se realizan desde dos puntos de vista principales: el desarrollo de procesos formativos adecuados y el marco de actuación en el que pueden realizar su actuación.

Para abordar estas cuestiones, Arandía (1997) recomienda crear un proceso formativo de iniciación que contemple tanto a los profesionales de nueva incorporación como a sus colaboradores, al tiempo que se instaure un proceso formativo continuo dirigido a todos los docentes con experiencia.

Tal y como señalan Marzo y Figueras (1990), existe una necesidad imperiosa de que el educador de adultos reciba una formación inicial que incluya una serie de conocimientos y competencias que faciliten la puesta en práctica profesional de las funciones del profesor o la profesora de adultos. Esta formación no puede ser cerrada, sino que ha de permitir la colaboración de los especialistas y la movilidad de la educación de adultos a la educación general.

Considerando la pluralidad de las funciones que tienen que desempeñar los educadores de adultos, se puede indicar que la formación que reciben actualmente los maestros en las facultades de Ciencias de la Educación no es suficiente como formación inicial para el profesorado de personas adultas, debido a que su orientación va más dirigida al público infantil o preadolescente. Las respuestas didácticas que allí se dan no son suficientes para la problemática cultural, social y económica de los adultos.

\section{La profesionalización del educador de personas adultas}

Históricamente, la función docente no siempre se consideró una profesión, y su estado de formación fue de abandono a lo largo de mucho tiempo. La situación laboral de los profesores no siempre gozó del mismo prestigio, los salarios no se correspondían con el trabajo realizado, la formación no era la 
adecuada, había un alto índice de feminización, etc. Todo ello conllevó que, socialmente, la labor docente fuera concebida como una semiprofesión.

Hoy en día, en ocasiones, se sigue cuestionando si la docencia es considerada una profesión o una ocupación. Este hecho no pasa desapercibido al cuerpo de educadores de adultos, ya que a ellos también se les cuestiona su profesionalidad.

El término profesionalización puede concebirse desde puntos de vista diferentes. Marzo y Figueras (1990) exponen cuatro componentes que definen el cumplimiento de una profesionalización:

- El rol social estará en relación con las funciones que se señalaron anteriormente y que debe asumir el educador.

- Las consecuencias sociales se configuran en la medida en que se asientan en los proyectos educativos en los que interviene como nuevas relaciones sociales.

- La remuneración, si se va estabilizando, procura la seguridad necesaria para seguir profesionalizándose por la práctica.

- Ser centro de interés principal en la vida está condicionado a las actitudes del educador y a la persistencia en el logro del reconocimiento de su estatus profesional.

Según Sáez (1996), nos encontraríamos con dos perspectivas: la social y la formativa. Desde la perspectiva social, la palabra profesionalización quiere indicar el reconocimiento social que tiene una ocupación. Por su parte, la perspectiva formativa se refiere al conjunto de conocimientos y de habilidades que necesitan los profesores para el buen ejercicio de su práctica educativa.

Tal y como señalan Sosa y Ribeiro (2015), la profesionalización del profesor es un proceso de formación y cambio que permitirá dar sentido y significado a su práctica docente.

Podemos afirmar que existe una clara tendencia ante la necesidad de profesionalizar a los educadores de personas adultas, con lo cual resulta necesaria la promoción de acciones académicas y organizativas para el desarrollo de este campo, a fin de lograr un reconocimiento social pleno y su consiguiente mejora en las condiciones de trabajo de los educadores de adultos.

\section{Metodología}

El objetivo principal del presente estudio fue identificar el perfil docente de los educadores de personas adultas, así como las percepciones que estos tienen sobre su labor docente, con el fin de averiguar si consideran que el trabajo que realizan es una profesión.

La recogida de la información se llevó a cabo a través de un instrumento elaborado con motivo de esta investigación, que perseguía obtener información acerca de las ideas que tienen los profesionales de la educación de adultos en relación con una serie de ítems asociados a distintas dimensiones 
sobre su labor docente. La descripción detallada de dicha escala se presenta a continuación.

\subsection{Muestra y procedimiento}

La población objeto de estudio estuvo constituida por los 196 profesionales de la educación que han impartido docencia en educación de adultos en la comunidad autónoma de Galicia a lo largo del curso 2013-2014, de los cuales interesa conocer su percepción sobre su labor docente. El procedimiento de muestreo fue no probabilístico, accidental o incidental, condicionado por la disponibilidad de los sujetos a participar en el estudio. Este tipo de muestreos, aunque no permiten «extraer muestras representativas de la población, sí facilitan el estudio cualitativo en profundidad del tema que interesa dentro de un contexto determinado» (Martínez, 2007: 56).

En total, participaron 90 docentes de ambos géneros $(54$ mujeres y 36 hombres), lo que supone un $45,92 \%$ del total de la población objeto de estudio. En cuanto a sus edades, el $46,67 \%$ de los participantes tenía entre 46 y 65 años; el 33,33\%, entre 36 y 45 años, y tan solo un $20 \%$ de la muestra tenía entre 25 y 35 años. Por titulaciones, todos los participantes eran licenciados y solo un 6,67\% de ellos había cursado un máster. En cuanto a la dedicación a la docencia, el $100 \%$ trabajaba a tiempo completo. Y en relación con el tiempo dedicado a desempeñar la labor de educadores de personas adultas, el 46,6\% llevaba más de 16 años dedicándose a ella; el 26,7\%, menos de 5 años, y el mismo porcentaje, entre 6 y 15 años.

El cuestionario se administró a los participantes a principios del curso académico 2013-2014 a través de correo electrónico, correo postal o personalmente. La participación fue voluntaria y se garantizó el anonimato y la confidencialidad de los datos obtenidos. Para responderlo, se les concedió el tiempo que ellos consideraron oportuno, pero recordándoles que esta información era necesaria para poder avanzar en la investigación. Una vez respondido el cuestionario, lo entregaron de la misma forma que lo recibieron.

\subsection{Instrumento de medida}

Se utilizó un cuestionario que nos permitió obtener una visión general del pensamiento docente sobre su labor educativa y, puesto que su cumplimentación es relativamente rápida, era esperable que hubiera un número elevado de sujetos que decidiera participar en el estudio.

Los apartados que incluyó el cuestionario se elaboraron en torno al objetivo que nos marcamos para esta investigación y, por supuesto, teniendo en cuenta toda la fundamentación teórica expuesta anteriormente. Este cuestionario fue revisado por 4 expertos en la materia, con el fin de validar su contenido y su inteligibilidad para el participante. Teniendo en cuenta las correcciones realizadas, fue necesario dar forma concreta a los ítems en un todo coherente y unificado, tanto en cuanto a su orden de presentación, formato y 
redacción como en cuanto a sus opciones y modalidades de respuesta. Una vez revisado y corregido, se aplicó el instrumento a una muestra piloto de cinco profesores, con la finalidad de detectar problemas de funcionamiento de los ítems antes de su aplicación a la totalidad de la muestra (Expósito, Navarro, Thoilliez y López, 2010), pero el resultado fue óptimo y no se necesitó realizar más cambios.

Finalmente, el cuestionario constó de dos partes. La primera de ellas recogió los datos de los participantes, así como de los destinatarios de la educación que imparten los docentes. En la segunda parte del cuestionario, se diseñó una escala tipo Likert, dando al entrevistado la posibilidad de valorar del 1 al 5 cada posible respuesta que se le ofreció (1: muy poco, 2: insuficiente, 3: suficiente, 4: bastante, 5: mucho) en cuanto a distintos aspectos relacionados con la educación de personas adultas. Así, el cuestionario quedó dividido en cinco dimensiones, las cuales pasamos a describir a continuación:

- Destinatarios de la educación: 11 ítems con los que se pretendió indagar a quién va dirigida la educación que imparten los encuestados (edad, género, nivel educativo, etc.).

- Desarrollo laboral: 14 ítems con los que se pretendió conocer lo que los entrevistados piensan de su labor como educadores de personas adultas.

- Formación: 22 ítems dirigidos a recoger información para conocer el grado de formación que poseen los educadores de adultos y su satisfacción con esa formación que poseen.

- Profesionalización: 13 ítems con los que se pretendió averiguar si los educadores de personas adultas consideran que su labor educativa es una profesión o no.

Para analizar los datos obtenidos en la dimensión «Destinatarios de la educación», se analizaron las respuestas de forma directa, agrupándolas en categorías en función de su similitud. Para analizar las respuestas aportadas por los docentes en el resto de las dimensiones («Desarrollo laboral», "Formación» y «Profesionalización»), incluidos también algunos ítems de la dimensión «Destinatarios de la educación", hallamos un valor medio de la escala Likert de 1 a 5 .

\section{Resultados}

A continuación, se exponen los resultados obtenidos en cada una de las dimensiones de las que se componía el cuestionario.

\subsection{Destinatarios de la educación}

El profesorado imparte docencia a alumnos de diferentes edades, desde los 16 años hasta más de 65, con lo que un mismo docente puede abarcar varias franjas de edad. El perfil del alumnado al que estos educadores de personas adultas imparten docencia (ver el gráfico 1) es el de unos estudiantes real- 
mente jóvenes. De hecho, la franja de edad con más alumnos es la de entre 18 y 25 años (un 100\%) y mayoritariamente del sector masculino (un $46,66 \%)$. También constatamos la presencia significativa de alumnos entre 16 y 18 años (un 46,67\%). Lo menos frecuente es la presencia de personas que superen los 65 años.

El ciclo o nivel prioritario en el que imparten clases los encuestados se corresponde con el de bachillerato (un 86,67\%), seguido de la educación secundaria de adultos, donde imparte clase un $53,33 \%$, y solo un $13,33 \%$ imparte su docencia en los ciclos formativos.

Según los encuestados, el principal motivo por el cual los alumnos de educación de personas adultas acuden a clase es para terminar los estudios (ver el gráfico 2). Este motivo se corresponde con la elección que los encuestados

Gráfico 1. Identificación de los destinatarios de la docencia impartida por los participantes

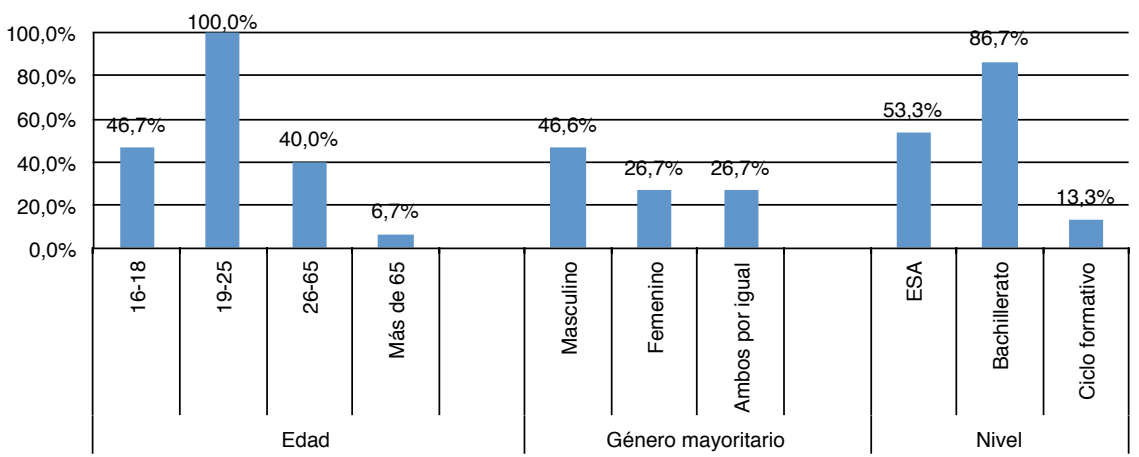

Fuente: elaboración propia.

Gráfico 2. Intereses de los alumnos según los docentes (valor medio en una escala Likert de 1 a 5)

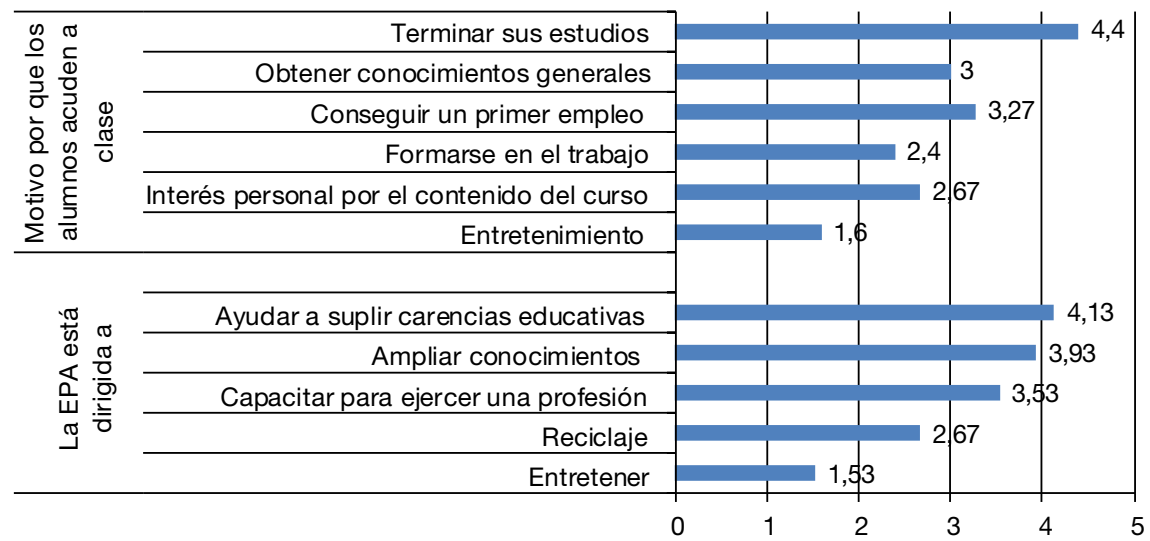

Fuente: elaboración propia. 
hacen ante la pregunta de hacia qué se dirige fundamentalmente la EPA, ya que la mayoría responde a "Ayudar a suplir carencias educativas" $\mathrm{O}$ "Ampliar conocimientos». El segundo motivo mayoritario por el cual los alumnos acuden a clase es para "Conseguir un primer empleo", lo cual se corresponde con la tercera opción que eligen sobre la función de la EPA: «Capacitar para ejercer una profesión».

\subsection{Desarrollo laboral}

La mayoría considera que es educador de personas adultas porque siente vocación por la labor que hace, mientras que otros llegaron a impartir docencia a este colectivo porque se encuentran sustituyendo a otros docentes (ver gráfico 3).

Gráfico 3. Desarrollo laboral de los participantes (valor medio en la escala Likert de 1 a 5

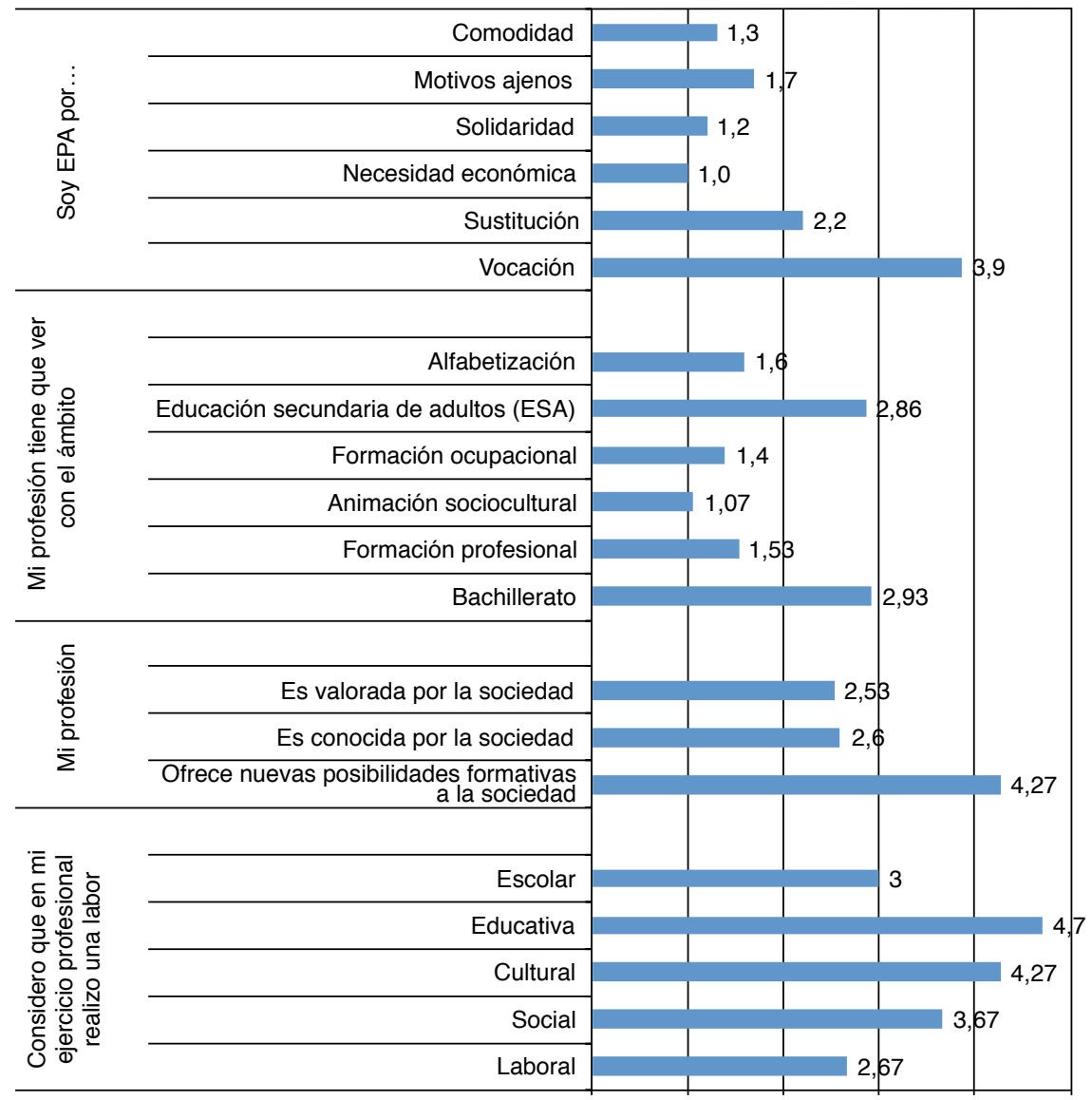

Fuente: elaboración propia. 
Por su parte, el ámbito profesional al que se dedica la mayoría de los educadores de personas adultas es el de bachillerato y educación secundaria de adultos. La mayoría de los encuestados consideran que su trabajo ofrece nuevas posibilidades formativas a la sociedad, al mismo tiempo que percibe que la sociedad ni conoce ni valora la labor que este grupo de profesionales realiza.

Teniendo en cuenta el ámbito profesional al que se dedica la mayoría del profesorado, este considera que la labor que fundamentalmente realiza en su ejercicio profesional es educativa, con un claro carácter compensador, como se pone de manifiesto cuando se le pregunta en el apartado «Destinatarios de la educación» sobre cuál es la principal tarea de la educación de personas adultas, y la mayoría manifiesta que es "Ayudar a suplir carencias educativas». Por otro lado, hay una gran cantidad de docentes que consideran que, en su ejercicio profesional, realizan una labor cultural e incluso social.

\subsection{Formación}

Parece claro que, para una gran mayoría de educadores de personas adultas, las cualidades que deben poseer para desarrollar su profesión están estrechamente relacionadas con el alumnado al que le van a impartir clase, ya que lo que más valoran es conocer cómo aprenden sus alumnos y comprender por qué las personas adultas acuden a sus clases (ver gráfico 4 ).

$Y$ aunque no tienen una opinión clara en cuanto a que sea un requisito imprescindible recibir formación específica para impartir educación a personas adultas, y otra mayoría coincide en estar de acuerdo en que poseen suficientes estudios para afrontar su trabajo, cuando se les pregunta si estarían dispuestos a ampliar sus conocimientos o su formación, en general, se muestran de acuerdo con este hecho, señalando que en lo que más les interesaría profundizar sería en cuestiones relativas a la metodología.

Resulta curioso que una gran mayoría de este colectivo apenas conoce bibliografía u otro tipo de información especializada en el tema ni tampoco sabe de centros que formen a educadores de personas adultas.

\subsection{Profesionalización}

Según los datos recogidos, la profesión de los educadores de personas adultas no está muy arraigada, ya que la mayoría se muestra indecisa a la hora de categorizar su labor como una profesión, incluso ellos mismos muestran dudas en cuanto a si se consideran o no educadores de personas adultas (ver el gráfico 5).

En general, suelen estar en desacuerdo con que exista una titulación específica de educadores de personas adultas. De hecho, opinan que no estarían más valorados si existiese dicha titulación.

Y, como se comentó con anterioridad, la mayoría considera que la sociedad ni conoce ni valora el trabajo que este grupo de profesionales realiza. Creencia por otra parte compartida con otras profesiones relacionadas con la educación a las que consideran insuficientemente valoradas. 
Gráfico 4. Necesidades formativas de los educadores de personas adultas (valor medio en una escala Likert de 1 a 5)

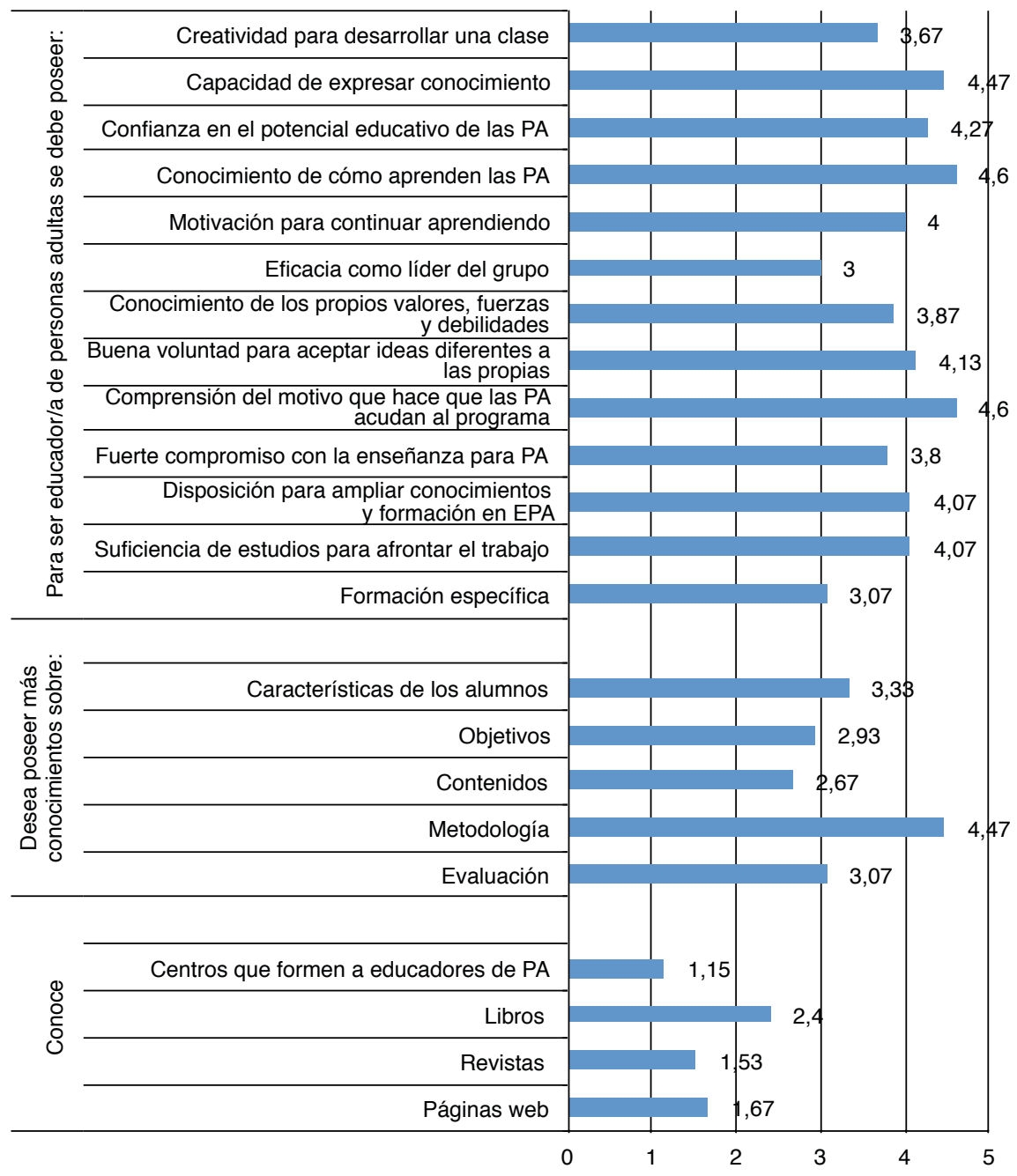

Fuente: elaboración propia.

En cuanto a las principales funciones que deberían de cumplir estos profesionales, la mayoría responde lo siguiente: «Estar dispuesto/a a seguir aprendiendo», «Transmitir conocimientos» y «Promover la participación». 
Gráfico 5. Profesionalización de los educadores de personas adultas (valor medio en una escala Likert de 1 a 5)

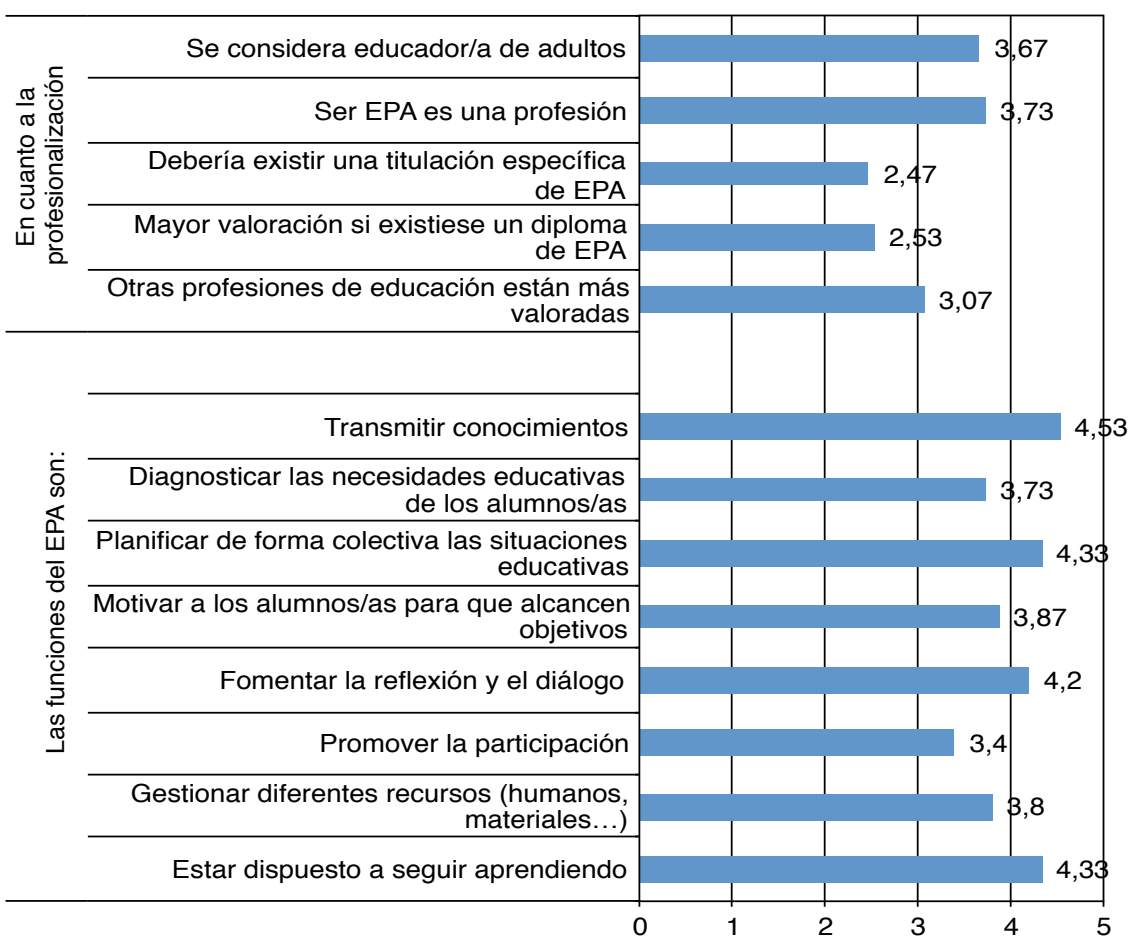

Fuente: elaboración propia.

\section{Conclusiones y discusión}

La educación de personas adultas es un campo que, a lo largo de la historia, ha estado ligado a la tarea de la alfabetización. Sin embargo, dadas las características de la sociedad actual, con sus avances, necesidades, intereses, etc., y teniendo en cuenta la investigación que hemos llevado a cabo, se ha comprobado que la educación de personas adultas ha dado un vuelco en cuanto a su concepción. Según Sáez (1996), hoy en día, este tipo de educación abarca nuevas dimensiones, ya que ayuda a suplir carencias educativas o a ampliar conocimientos. De hecho, en el nivel educativo donde acuden más alumnos es en el bachillerato, y además la edad de los educandos varía entre los 16 y los 65 años, es decir, no se circunscribe a un grupo de edad determinado.

Este tipo de educación es la que se dirige a un colectivo particular: las personas adultas, un público heterogéneo caracterizado por su gran diversidad cultural, social, intelectual, experiencia, nivel económico, necesidades, edad, etc. Asimismo, consideramos que la educación de personas adultas se funda- 
menta en un criterio aplicable a otros terrenos pedagógicos, como es el de la formación permanente y continuada (González, 2010), que tiene como finalidad enriquecer las capacidades y las habilidades, ampliar los conocimientos y mejorar las competencias técnico-profesionales del colectivo al que se dirige, favoreciendo su desarrollo personal, facilitando su acceso a los bienes culturales y aumentando su bienestar, de manera que pueda participar más activamente en la sociedad.

En nuestra opinión, la educación es un proceso que nunca debería dejar de lado dos ideas clave muy relacionadas: la primera de ellas es que la educación debe ser con y para las personas, es decir, tiene que desarrollarse teniendo en cuenta a todos los agentes implicados (profesorado, educando, comunidad y entorno) y todos deben participar activamente en el desarrollo del discurso educativo. La segunda idea haría referencia a que la educación debe ser un proceso multidireccional: de docentes a educandos, de educandos a docentes, de sociedad a escuela y de escuela a sociedad.

Creemos que ambas ideas serían garantía de un aprendizaje colaborativo y significativo en el que todos participamos trabajando en grupos heterogéneos en proyectos diversos (investigación, Administración, etc.), con la finalidad de ser personas de provecho que interactúan en una sociedad que avanza a pasos agigantados.

Pero este hecho contrasta con lo que se puede constatar en nuestra investigación, ya que los educadores de personas adultas manifiestan que ellos realizan una buena labor de cara a la sociedad, pero, por el contrario, esta no responde de forma positiva a lo que ellos ofrecen, puesto que no se sienten valorados ni se conoce el trabajo que realizan. Por lo tanto, si la sociedad no se implica más en este campo de la educación, esta nunca le asignará la importancia que le corresponde, ni se podrá pretender que existan avances en esta área.

Consideramos que los educadores de personas adultas tienen como objetivo principal reconocer que están ante un colectivo con personalidad y características propias, de tal manera que pueden orientar, adaptar y facilitar sus aprendizajes a través de la detección y el diagnóstico de las necesidades socioeducativas que presente, con miras a planificar de forma colectiva, cooperativa y democrática situaciones didácticas reales, a través del diálogo, la reflexión y un espíritu crítico, lo cual favorecerá su autonomía y su independencia, además de la toma de decisiones sobre los hechos en los que participa.

Aunque los profesores de personas adultas consideran, en cuanto a la formación, que su función principal es seguir aprendiendo, también opinan que no es requisito imprescindible el recibir formación específica para impartir educación a dicho segmento de edad. Este hecho conlleva que, actualmente, en los centros que se dedican a dicho ámbito, las clases sean impartidas por educadores que no poseen una formación específica sobre el mundo adulto (Rose et al., 2005) que les permita desarrollar una práctica pedagógica de calidad y adecuada al colectivo al que se dirigen. Sin embargo, esta formación sí existe en el caso de otros colectivos, puesto que tanto en educación infantil como en primaria se recibe formación específica adecuada al nivel y a las características del alumnado. Este 
dato demuestra que, en realidad, la no formación específica de los profesores obedece a la ausencia de requisitos de esta formación para el desempeño en los centros de EPA por parte de la Administración educativa.

Debemos plantearnos ahora el tema de la profesionalización de la educación de personas adultas, una demanda que empieza a asentarse en algunos países de nuestro entorno y también en el nuestro.

Para situar mejor esta realidad, cabe recordar que la palabra profesionalización indica el reconocimiento social que tiene una ocupación (Sáez, 1996). Por lo tanto, cualquier docente que esté ejerciendo la educación de personas adultas y tenga un reconocimiento profesional debe ser un profesional. Autores como Sosa y Ribeiro (2015) consideran que la profesionalización del enseñante es un proceso de formación y de cambio que permitirá dar sentido y significado a su práctica docente, y es ahí donde se encuentran las carencias de este colectivo, pues no disponen de formación específica al respecto.

Aunque el colectivo de educación de personas adultas, en su aspiración por la profesionalización, ha venido trabajando diversas áreas que permiten ubicar su trabajo como una profesión (participación en diferentes asociaciones profesionales, divulgación de artículos e investigaciones en publicaciones especializadas, búsqueda de fuentes de información, además de la creación de estudios de postgrado y acreditaciones), resulta curioso que, en nuestra investigación, los docentes consultados que consideran que ser educador de personas adultas es una profesión representan casi el mismo número de los que expresan que no lo es. Además, una amplia mayoría de los mismos considera que no es necesario que exista una titulación específica de EPA y, si existiera, opinan que ello no haría que la sociedad valorase más la labor que realizan.

Teniendo en cuenta la carencia de formación específica y la profesionalización de los educadores, hay que comentar que no pretendemos dar a entender que haga falta una especialidad en educación de personas adultas, pero sí defendemos la necesidad de una formación que les permita adaptarse a las características, a las necesidades y a los intereses de dicho sector de población. Es por ello que reconocemos la necesidad de que exista una formación inicial del educador de personas adultas con conocimientos y competencias que faciliten la puesta en práctica profesional de sus funciones, una formación con un carácter específico, tal y como señala Morón (2014), que requiere un enfoque diferente, tanto en el planteamiento conceptual como en el procedimental o metodológico. Así, «la formación universitaria debe ser capaz de conjugar la adquisición de saberes propios a la disciplina ligada a la profesión y organizar los espacios necesarios para la manifestación de las competencias requeridas al ejercicio de esa profesión» (Correa, 2015: 261).

Pero, además, y a la luz de los datos de esta pequeña investigación realizada, pensamos que también es importante que exista una formación de los profesores en ejercicio. Para ello, quienes participen en el ámbito educativo que hemos tratado aquí deben comprometerse a actualizar y redefinir su propia práctica, teniendo en cuenta las teorías que ha ido construyendo este campo de conocimiento. 
Para finalizar, no debemos olvidar que la sociedad actual y el mundo competitivo en el que vivimos exigen una acreditación para acceder al mundo del trabajo, una certificación que ubique al sujeto como una persona con plenas facultades para desempeñar una actividad o un cargo determinados. De esta forma, «la educación de las personas adultas deja de ser un proyecto de emancipación y cambio social para convertirse en servidora del mercado, con la finalidad de mejorar las destrezas para incrementar el trabajo productivo y aumentar la competitividad» (Rumbo, 2013: 182). Consiguientemente, pensamos que los educadores de personas adultas podrían, en un futuro no muy lejano, tener esta acreditación que los cualificara como tales, no solo como requisito para acceder al mercado laboral, sino también como garantía de mejora de la calidad de la enseñanza que deben recibir y brindar.

\section{Referencias bibliográficas}

ARANDía, M. (1997). Los educadores de personas adultas y su formación ante un mundo de cambio. En M. J. Cabello (coord.). Didáctica y Educación de Personas Adultas: Una Propuesta para el Desarrollo Curricular (pp. 131-154). Málaga: Aljibe.

ArbuÉs, M. y Tarín, L. (2000). Aprender a lo largo de la vida y las nuevas tecnologías. En J. Duart y A. Sangrá (eds.). Aprender en la virtualidad (pp. 51-60). Barcelona: Gedisa.

Correa, E. (2015). La alternancia en la formación inicial docente: Vía de profesionalización. Educar, 51(2), 251-275. $<$ https://doi.org/10.5565/rev/educar.712>

Expósito, E.; Navarro, E.; Thoilliez, B. y López, E. (2010). Determinants of child well-being: A perspective from students of education. Comunicación presentada en la European Conference on Educational Research (ECER). Helsinki, 23 a 27 de agosto.

GonzÁlez, J.L. (2010). Hacia dónde va la educación de las personas adultas. EduPsycho, 2, 29-32.

Ley $1 / 1990$, de 3 de octubre, de ordenación general del sistema educativo (LOGSE). Boletín Oficial del Estado (4 octubre 1990), núm. 238, p. 28927-28942.

Ley Orgánica 2/2006, de 3 de mayo, de educación (LOE). Boletín Oficial del Estado (4 mayo 2006), núm. 106, p. 17158-17207.

Ley Orgánica 8/2013, de 9 de diciembre, para la mejora de la calidad educativa (LOMCE). Boletín Oficial del Estado (10 diciembre 2013), núm. 295, p. 97858 97921.

MarTínez, R.A. (2007). La investigación en la práctica educativa: Guía metodológica de investigación para el diagnóstico y la evaluación en los centros docentes. Madrid: Ministerio de Educación y Ciencia.

Marzo, A. y Figueras, J. (1990). Educación de adultos: Situación actual y perspectivas. Barcelona: ICE / HORSORI.

Morón, J. (2014). Educación y personas mayores. Revista Electrónica Interuniversitaria de Formación del Profesorado, 17(1), 107-121. $<$ https://doi.org/10.6018/reifop.17.1.198871>

Rose, A.; Jeris, L. y Smith, R. (2005). Is Adult Education a Calling?: Shaping Identity and Practice in Steel Mill Learning Centers. Teachers College Record, Columbia University, 107(6), 1305-1334. 
Rumbo, B. (2010). La profesionalización de la educación de personas adultas. Revista Iberoamericana de Educación, 54(4), 1-7.

- (2013). Tensiones y dilemas en la educación de las personas adultas. Teoría de la Educación y Cultura en la Sociedad de la Información, 14(2), 178-191.

- (2016). Problemas y retos de la educación de las personas adultas. Educar, 52(1), 93-106. $<$ https://doi.org/10.5565/rev/educar.707>

SÁEz, J. (1996). La profesionalización de los educadores sociales: Algunas consideraciones introductorias. En J.A. López (coord.). El educador social: Lineas de formación y de actuación (pp. 11-21). Madrid: Guillermo Mirecki.

Savelli, S. (2014). Teaching and learning in the society of continuing education and training. Universal Journal of Educational Research, 2(5), 414-420. <https://doi.org/10.13189/ujer.2014.020503>

Sosa, L. y Ribeiro, C. (2015). El conocimiento profesional como característica distintiva de profesionalización docente en la formación de profesores. Revista Iberoamericana de Producción Académica y Gestión Educativa, 2, 1-19.

Watkins, K. y Marsick, V. (2014). Adult education \& human resource development: Overlapping and disparate fields. New Horizons in Adult Education and Human Resource Development, 26(1), 42-54.

<https://doi.org/10.1002/nha3.20052>

ZabalzA, M. (2002). La enseñanza universitaria: El escenario y sus protagonistas. Madrid: Narcea. 